International Journal of Applied Chemistry.

ISSN 0973-1792 Volume 17, Number 1 (2021) pp. 41-53

(C) Research India Publications

https://dx.doi.org/10.37622/IJAC/17.1.2021.41-53

\title{
UV, FTIR and DFT studies of Pyrazolines in polar protic and polar aprotic solvent mixtures
}

\author{
Walmik B. Shirsath \\ Department of Chemistry, Karm.A.M. Patil Arts, Comm. and Kai Annasaheb N.K. \\ Patil Science Senior College, Pimpalner. Tal. Sakri, Dist. Dhule. 424306, \\ Maharashtra, India.
}

\begin{abstract}
UV and FTIR spectroscopic studies containing 0.01 and $0.001 \mathrm{~m}$ some Pyrazolines with electron donating and withdrawing groups were done in $10-90 \%(\mathrm{w} / \mathrm{w})$ methanol (ME) in DMSO/DMF solvent mixtures at room temperature. The $\lambda_{\max }$ of solutes vary with the \% of ME. The decrease was more in ME + DMSO than in ME + DMF. The amount of blue shift (lower wave length) has been used as a measure to the strength of hydrogen bond. Absence of $n \rightarrow \pi^{*}$ excitation in solute $+\mathrm{ME}+$ $\mathrm{DMSO} / \mathrm{DMF}$ was ascribed due to hydrogen bond formation. An irregular trends in $v \mathrm{OH}, v \mathrm{C}=\mathrm{O}, v \mathrm{~S}=\mathrm{O}, v \mathrm{C}-\mathrm{Cl}, v \mathrm{C}-\mathrm{H}, v \mathrm{NH}$ etc. with increase of $\mathrm{ME}$ in solution at a particular concentration of solute indicated structure of the medium changed with $\mathrm{ME}$ content. In certain compositions of 0.01 and $0.01 \mathrm{~m}$, no changes in vibration frequencies were detected. $v \mathrm{OH} / \mathrm{vNH}$ for Pyrazolines in both concentrations $(0.01$ and $0.001 \mathrm{~m}$ ) in all ME + DMF are greater than those of ME + DMSO, thereby indicating possibility of intramolecular hydrogen within Pyrazolines and intermolecular hydrogen bond among solvent molecule Hydrogen bond formation of $\mathrm{ME}$ with $\mathrm{C}-\mathrm{Cl}$ of Pyrazolines was well supported by shifting of normal $\mathrm{vC}=\mathrm{O}$ to $1650-1640 \mathrm{~cm}^{-1}$. Geometry optimizations of heterocyclic compounds, pure solvents and their $1: 1$ and 1:1:1 complex were carried out using the DFT/B3LYP method with medium size 6$31 \mathrm{G}(\mathrm{d})$ basis set available in G03(W) series of programs All optimized geometries were viewed through Gauss View 4.1 software to know exact nature of intermolecular interaction.
\end{abstract}

Keywords: UV, FTIR, DFT and molecular interaction

\section{INTRODUCTION}

Pyrazolines have played a crucial part in the development of theory in heterocyclic chemistry. Pyrazolines are well known important nitrogen containing five member 
heterocyclic compounds. Increasing evidence suggest that pyrazoline derivatives possess a broad spectrum of biological activities such as tranquillizing, muscle relaxant, antidepressant, anticonvulsant, psycho analeptic, anti hypotensive antibacterial ,antifungal, antitubarcular, antitumor, antidepressant insecticidal, antidiabetic [1-5]. Pyrazolines are used extensively as synthons in organic synthesis reports [6]. The synthesis [7] of $\alpha, \beta$-unsaturated carbonyl compounds is one of main structural component in various naturally occurring and biologically essential substance. Several strategies for the synthesis of these system based on formation of carbon-carbon bond have been reported and among them the direct Aldolcondensation and Claisen-Schimdt condensation still occupy prominent position It is well known that most natural or synthetic chalcones are highly active with extensive pharmaceutical and medicinal application. Chalcones are found to be effective as antiviral cardiovascular and anti-inflammatory agents. Reaction of 2'-hydroxy5 'acetamido chalcones with catalytic amount of iodine in DMSO affords flavones molar amount of mercuric (II) acetate in pyridine gives aurones while with phenylhydrazine and hydrazine hydrate gives pyrazoline in triethanolamine medium [8].

Pyrazolines constitute a medicinal important class of heterocyclic small molecules that have shown potential bioactivity in numerous screening tests. Pyrazolines systems are known to be biologically active and are important constituents of many pharmaceutical and agrochemical products on the synthesis and pharmacology of pyrazolines revealed that some of these compounds exhibited a wide local anesthetic activity. Therefore, the synthesis of these compounds was of interest largely on account of their biological activity. Pyrazolines are important nitrogen containing heterocycles possessing diverse biological activities such as anticarcinogenic, antidiabetic, anticonvulsant, anti-inflammatory, antifungal, antiviral, and analgesic and antioxidant activities1, 3,5-Trisubstituted pyrazolines represent a very important class of biologically active agents and the focus of a significant amount of research interest. In particular, pyrazolines derivatives have found use as antitumor, antibacterial, antifungal, antiviral, antiparasitic, antitubercular and insecticidal agents Pyrazolines are known for their pronounced antimicrobial activity. Pyrazolines which contain furobenzopyrone or benzofuran moieties are well known to possess broad spectrum anti-inflamatory activity The new pyrazolines and isoxazoles containing methylthio phenyl group in position 3 and aryl groups in position 5 and evaluated there in vivo analgesic and anti-inflammatory properties [9-12].

Ultra violet spectroscopy is also known as electronic spectroscopy. It involves the promotion of electron ( $\sigma, \pi$ and $n$ electrons ) from the ground state to higher energy state. It is very useful to measure the number of conjugated double bonds and also aromatic conjugation within the various molecules [13]. The solvent effect on spectra resulting from electronic transition is primarily dependent on the chromophore and the nature of transition ( $\Pi^{-} \Pi^{*}$ and $n-\Pi^{*}$ transition. $\sigma \rightarrow \sigma^{*}, n \rightarrow \sigma^{*}, \Pi \rightarrow \Pi^{*}, n \rightarrow \Pi^{*}$ and charge transfer absorption). IR spectroscopy is a common technique that is used to identify the major functional group in a compound. The identification of these groups depends upon the amount of IR radiation absorbed and the particular frequency at 
which these groups absorb. It is the leading method for identification of hydrogen bonds. For instance the formation of an O-H--O hydrogen bond elongates and weakens the $\mathrm{O}-\mathrm{H}$ bond. The resulting red shift of the $\mathrm{O}-\mathrm{H}$ bond stretching frequency can be easily detected and its magnitude indicates the strength of the hydrogen bond. IR spectrum measure the degree of the molecular vibration and rotation of the bonds in the molecule. Each type of the functionality will absorb at certain characteristics frequency and with characteristic strength. IR spectrum is an important record which gives sufficient information about the structure of a compound [14]. IR spectroscopy is used to establish whether a given sample of an organic substance is identical with another or not. This is because large numbers of absorption bands are observed in the IR spectra of organic molecules and the probability that any two compounds will produce identical spectra is almost zero. So if two compounds have identical IR spectra, then both of them must be samples of the same substances.

\section{EXPERIMENTAL SECTION}

Aqueous $\mathrm{KOH}(0.02 \mathrm{~mol})$ was added to the suspension of 2-hydroxyacetophenone $(0.01 \mathrm{~mol})$ and $\mathrm{p}$-chlorobenzaldehyde $(0.01 \mathrm{~mol})$ in ethanol. The reaction mixture was stirred at room temperature for overnight. The reaction mixture was then poured onto ice cold water and acidified with $\mathrm{HCl}(1 \mathrm{M})$. The solid obtained was filtered and crystallized from ethanol to get pure Chalcone 1. It's structural formula shown in figure (1).<smiles>O=C(/C=C/c1ccc(Cl)cc1)c1ccccc1O</smiles>

Figure 1: Chalcone 1:(2E)-3-(4-chlorophenyl)-1-(2-hydroxyphenyl)prop-2-en-1-one

Chalcone $1(0.002 \mathrm{~mol})$ was dissolved in ethanol $(10 \mathrm{ml})$. To this reaction mixture, hydrazine hydrate $(0.006 \mathrm{~mol})$ was added. The reaction mixture was heated under mild reflux for $2.5 \mathrm{hrs}$. After completion of the reaction (monitored by TLC), the reaction mixture was cooled to room temperature and poured onto ice cold water. The white solid obtained was filtered off and crystallized from ethanol to obtain pure pyrazoline $\left(\mathrm{P}_{1}\right)$ and further purified by column chromatography using hexane ethyl acetate $(80: 20 \mathrm{v} / \mathrm{v})$ as an eluent. Figure 2 indicates Pyrazoline $\left(\mathrm{P}_{1}\right)$ as shown below 
<smiles>Oc1ccccc1C1=NNC(c2ccc(Cl)cc2)C1</smiles>

Figure 2: Pyrazoline $\left(\mathrm{P}_{1}\right)$ : 2-[5-(4-chlorophenyl)-4, 5-dihydro-1H-pyrazol-3-yl] phenol

Same procedure was followed to synthesize Chalcone 2 from m-nitrobanzaldehyde. Similarly $\mathrm{P}_{2}$ as shown in figure 3 was prepared by using Chalcone 2 .<smiles>O=[N+]([O-])c1cccc(C2CC(c3ccccc3O)=NN2)c1</smiles>

Figure 3: Pyrazoline $\left(\mathrm{P}_{2}\right)$ : 2-[5-(3-nitrophenyl)-4, 5-dihydro-1H-pyrazol-3-yl] phenol

All chemicals were of standard grade. Binary mixtures were prepared by mixing a known mass of each liquid. Important synthesized heterocyclic compounds of pyrazoline were used. The solids were characterized by chemical as well as by spectroscopic methods. Binary liquid mixtures .of DMSO and DMF with ME( $\left.\mathrm{x}_{1}\right)$ of compositions $0-100$ mass $\%$ as well as $\mathrm{x}_{1}=0.1$ to 0.9 were prepared by mixing a known mass of each liquid in an airtight Stoppard bottle. Solutions containing 0.001 to $0.01 \mathrm{~m}$ hetrocyclies $\left(\mathrm{P}_{1}\right.$ and $\left.\mathrm{P}_{2}\right)$ in binary mixtures (as a solvent) were also prepared by usual method.

UV measurements: UV Spectra of above solvent systems and solutions were recorded on a UV $2400 \mathrm{PC}$ in the wavelength range $200-400 \mathrm{~nm}$. The scan speed was fast and sampling interval was about 2.0. The UV measurements are reported directly in $\mathrm{nm}$, and the corresponding range in $\mathrm{cm}^{-1}$.

\section{IR measurements}

FTIR spectra of experimental solvent systems and solutions were recorded on a FTIR spectrometer (Model: SIMADZU 8400S PC) by using KBr pellet in the region 400$4000 \mathrm{~cm}^{-1}$ with $4.0 \mathrm{~cm}^{-1}$ resolution. Selected stretching frequencies of solvents and solutes used in this work are listed in Table 1 and 2. 
Table 1: Selected neat IR frequencies of solvents

\begin{tabular}{|c|c|c|c|c|c|}
\hline \multicolumn{2}{|c|}{ ME } & \multicolumn{2}{c|}{ DMSO } & \multicolumn{2}{c|}{ DMF } \\
\hline Group & $v$ & Group & $v$ & Group & $v$ \\
\hline O-H & 3570,3450 & S=O & 1051.19 & C=O & 1752 \\
\hline C-H & 2833.00 & C-H & 2995,2963 & C-H & 2944.35 \\
\hline C-H & 2945.40 & -- & -- & -- & -- \\
\hline C-O & 1030.02 & -- & -- & -- & -- \\
\hline
\end{tabular}

Table 2: IR stretching frequencies of P1 and P2

\begin{tabular}{|c|c|c|c|}
\hline \multicolumn{2}{|c|}{ P1 $_{1}$} & \multicolumn{2}{c|}{ P $^{2}$} \\
\hline Group & $v$ & Group & 3401 \\
\hline N-H & 3339 & N-H & O \\
\hline O-H & 3024,3134 & O-H & $3292-2792$ (broad) \\
\hline C=N & 1591 & C=N & 1574 \\
\hline C=C & 1618 & C=C & 1614,1478 \\
\hline C-Cl & 741 & N=O & 1346,1525 \\
\hline
\end{tabular}

\section{Optimization of geometries using Gaussian Package}

Geometry optimizations of heterocyclic compounds, pure solvents and their 1:1 and 1:1:1 complexes were carried out using the DFT/B3LYP method with medium size 6$31 \mathrm{G}$ (d) basis set available in G03(W) series of programs [15]. All optimized geometries were viewed through Gauss View 4.1 software. Figure 4-6 includes optimized geometries of solvents and solutes under investigation. 

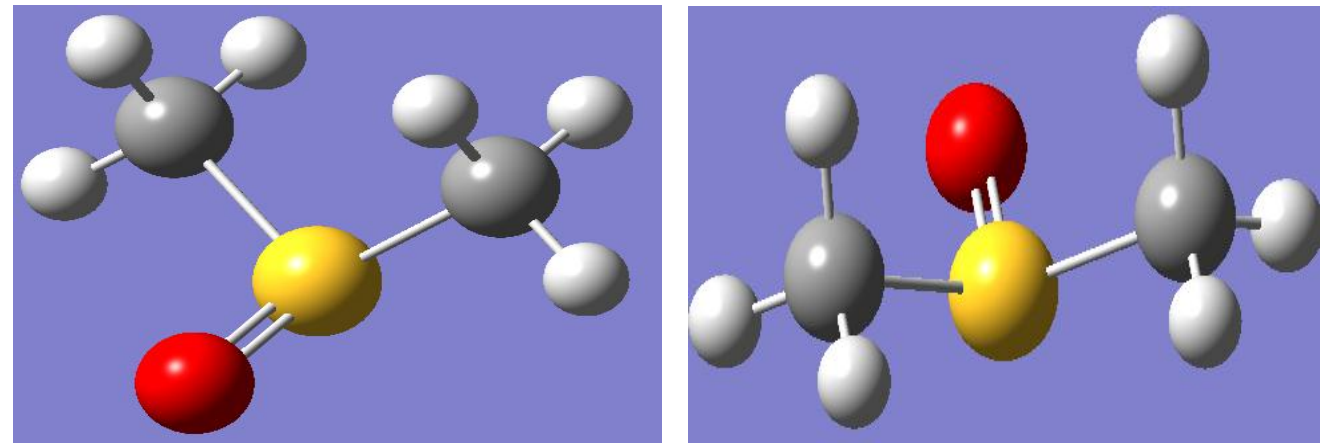

(a)
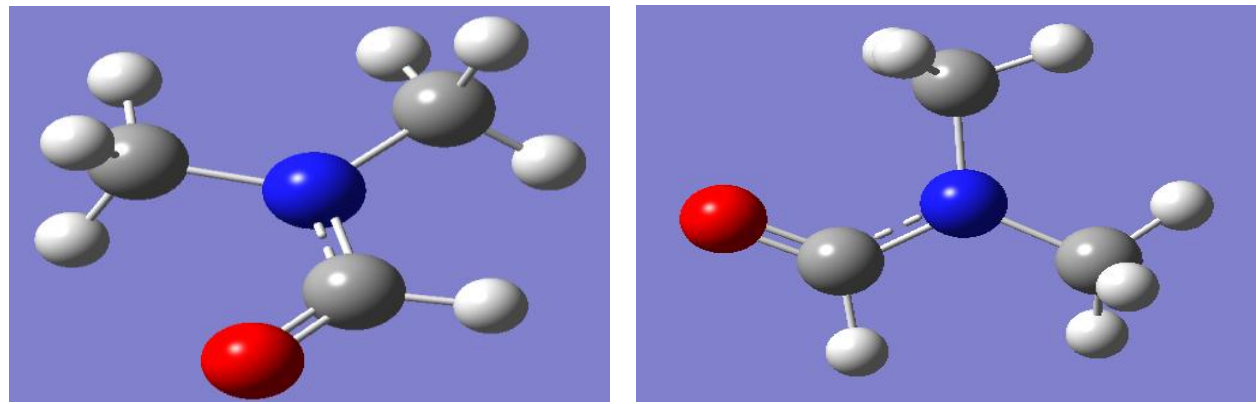

(b)

Figure 4: (a) Optimized geometries of DMSO using B3LYP/6-31G (d) basis set (b) optimized geometries of DMF using B3LYP/6-31G (d) basis set

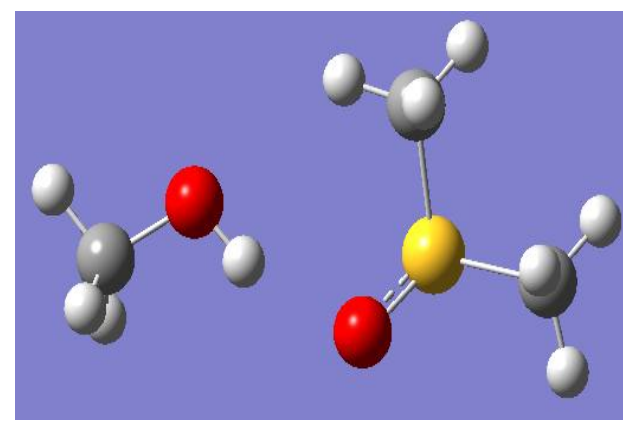

(a)

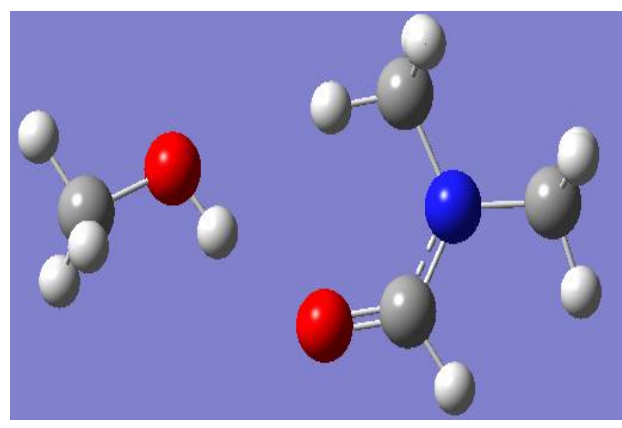

(b)

Figure 5: (a) Optimized geometry of Hydrogen bond interactions: ME with DMSO (b) Optimized geometry of Hydrogen bond interactions: ME with DMF 


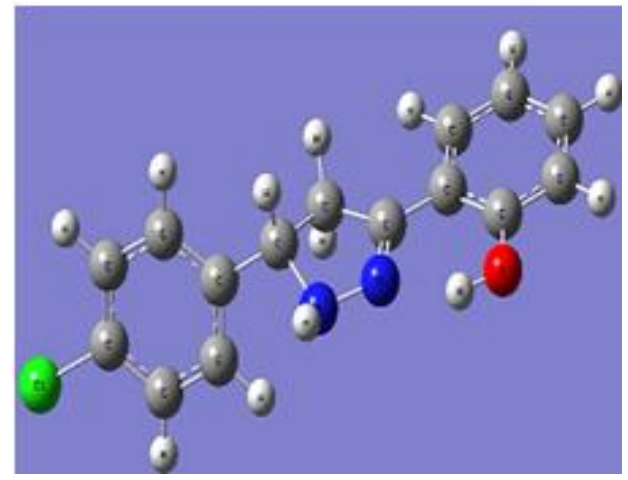

(a)

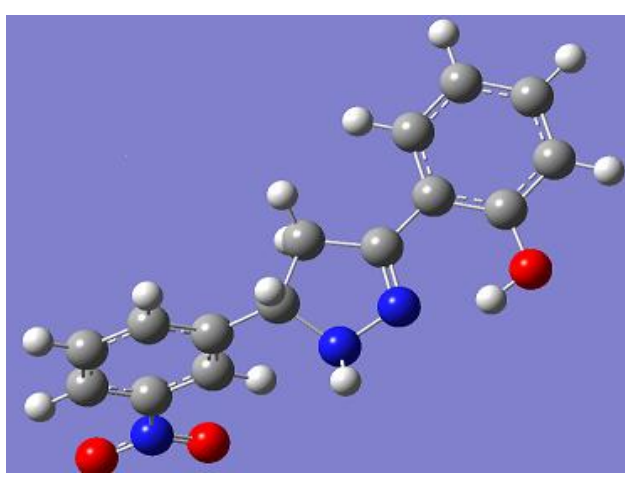

(b)

Figure 6: (a) DFT optimized geometries of $\mathrm{P}_{1}$ by using B3LYP/ 6-31G (d) basis set (b) DFT optimized geometries of $\mathrm{P}_{2}$ by using B3LYP/ 6-31G (d) basis set

\section{RESULT AND DISCUSSION}

\section{IR and theoretical studies}

Experimental results of FTIR stretching frequencies of some functional groups of mixtures of ME with DMSO and DMF are listed in Table 3. For ME + DMSO, maximum $\mathrm{vOH}\left(\right.$ at low $\left.\mathrm{x}_{1}\right)$ decreased upon dilution $\left(\mathrm{x}_{1} \approx 0.2-0.9\right)$. The magnitude of all these frequencies is higher than that for pure ME. This may be due to the breaking of ME aggregates making more free monomers of it in the system. The $v \mathrm{~S}=\mathrm{O}$ of DMSO disappeared at $1051.19 \mathrm{~cm}^{-1}$ in all compositions and appeared at a lower but almost constant value suggesting hetero association between ME and DMSO. In case of $\mathrm{ME}+\mathrm{DMF}$, both $v \mathrm{OH}$ and $\nu \mathrm{C}=\mathrm{O}$ decreased with the increase in the amount of $\mathrm{x}_{1}$ in the binary system suggesting breaking of aggregates of ME as well as DMF. An aldehyde $v \mathrm{C}-\mathrm{H}$ at $2944 \mathrm{~cm}^{-1}$ moved down upon addition of co- solvent indicating decreased double bond character of $\mathrm{C}=\mathrm{O}$ in presence of ME. The hydrogen bond formation between hydroxyl proton of $\mathrm{ME}$ and oxygen of $\mathrm{O}=\mathrm{S}$ (DMSO) / $\mathrm{O}=\mathrm{C}$ (DMF) is further confirmed by the theoretical investigation. Figure 5 depicts minimum energy structures for 1:1 complex of components of binaries using our legal licensed copy G03 (W) software. From above studies, hetero intermolecular interactions are in the following order: $\mathrm{ME}+\mathrm{DMSO}>\mathrm{ME}+\mathrm{DMF}$

IR, UV and theoretical studies Table 4 shows $v \mathrm{OH} / v \mathrm{NH}, v \mathrm{~S}=\mathrm{O}$ and $v \mathrm{C}-\mathrm{Cl}$ frequencies for $(0.01$ and $0.001 \mathrm{~m}) \mathrm{P} 1+\mathrm{ME}+\mathrm{DMSO}$. Distinguish between $v \mathrm{OH}$ and $v \mathrm{NH}$ is generally difficult because these frequencies appear in the same region (3500 $-3300 \mathrm{~cm}^{-1}$ ). An inspection of data in Table 4 shows that in DMSO, $v \mathrm{NH}$ is more free whereas $\mathrm{P}_{1}$ forms hydrogen bond with acidic hydrogen of $\mathrm{ME}$ and not much concentration effect of it upto $30 \% \mathrm{ME}$ was recorded. A maximum for this particular frequency was at $40 \% \mathrm{ME}$. After this composition, $v \mathrm{OH} / v \mathrm{NH}$ gets lowered with successive addition of $\mathrm{ME}$. The $\mathrm{vS}=\mathrm{O}$ remain indeed constant in position after $40 \% \mathrm{ME}$ indicating homo associated species gets converted into hetero associated 
species which are not interacting with $\mathrm{vOH}$ possibly due to intramolecular hydrogen bond in $\mathrm{P}_{1}$. Concentration effect for $\mathrm{vS}=\mathrm{O}$ was also null after this composition of polar protic solvent. The $v \mathrm{C}-\mathrm{Cl}$ in all compositions at both $\mathrm{P}_{1}$ concentrations had very little effect. Small $\vee \mathrm{C}-\mathrm{Cl}$ of the solute in pure $\mathrm{ME}\left(\approx 659 \mathrm{~cm}^{-1}\right)$ as compared to that in pure DMSO $\left(\approx 698 \mathrm{~cm}^{-1}\right)$ shows the complex formation between $\mathrm{P}_{1}$ and ME through (C-Cl of $\mathrm{P}_{1}$ and $\mathrm{H}-\mathrm{O}$ of $\mathrm{ME}$ ) hydrogen bond. In short for this system, we conclude that media prefer to interact with other parts of the solute rather with $v \mathrm{NH}$.

Table 3: Selected IR frequencies for binaries

$\operatorname{ME}\left(\mathrm{x}_{1}\right)+\operatorname{DMSO}\left(1-\mathrm{x}_{1}\right)$

\begin{tabular}{|c|c|c|}
\hline $\mathbf{x} 1$ & $\mathbf{O H}$ & $\mathbf{S}=\mathbf{O}$ \\
\hline 0 & -- & 1051.19 \\
\hline 0.1 & 3387.11 & 1031.95 \\
\hline 0.2 & 3381.33 & 1031.95 \\
\hline 0.3 & 3379.4 & 1031.95 \\
\hline 0.4 & 3379.4 & 1030.02 \\
\hline 0.5 & 3377.47 & 1030.02 \\
\hline 0.6 & 3373.61 & 1030.02 \\
\hline 0.7 & 3358.18 & 1030.02 \\
\hline 0.8 & 3346.61 & 1030.02 \\
\hline 0.9 & 3346.61 & 1030.02 \\
\hline 1 & 3352.39 & - \\
\hline
\end{tabular}

$\operatorname{ME}\left(\mathrm{x}_{1}\right)+\operatorname{DMF}\left(1-\mathrm{x}_{1}\right)$

\begin{tabular}{|c|c|c|c|}
\hline $\mathbf{x 1}$ & $\mathbf{O H}$ & $\mathbf{C = O}$ & $\begin{array}{c}\text { C-H } \\
\text { (Aldehyde) }\end{array}$ \\
\hline 0 & -- & 1675.00 & 2944.35 \\
\hline 0.1 & 3458.48 & 1674.27 & 2931.90 \\
\hline 0.2 & 3439.19 & 1674.27 & 2933.83 \\
\hline 0.3 & 3433.41 & 1672.69 & 2935.76 \\
\hline 0.4 & 3419.9 & 1672.34 & 2935.76 \\
\hline 0.5 & 3421.83 & 1664.62 & 2935.76 \\
\hline 0.6 & 3387.11 & 1668.48 & 2939.61 \\
\hline 0.7 & 3365.9 & 1668.48 & 2941.54 \\
\hline 0.8 & 3352.39 & 1670.41 & 2943.47 \\
\hline 0.9 & 3352.39 & 1648.48 & 2943.47 \\
\hline 1 & 3352.39 & -- & -- \\
\hline
\end{tabular}

The $v \mathrm{OH} / v \mathrm{NH}, v \mathrm{C}=\mathrm{O}, v \mathrm{C}-\mathrm{Cl}$ and $v \mathrm{C}-\mathrm{N}$ for $\mathrm{P}_{1}+\mathrm{ME}+\mathrm{DMF}$ are collected in Table 5 . All $v \mathrm{OH} / v \mathrm{NH}$ for $\mathrm{P}_{1}$ in both concentrations $(0.01$ and $0.001 \mathrm{~m})$ and in all ME + DMF are greater than those of $\mathrm{P}_{1}+\mathrm{ME}+\mathrm{DMSO}$. For a particular concentration of $\mathrm{P}_{1}$, they dropped with increase in ME content. After $50 \% \mathrm{ME}$, two sharp peaks were detected for $v \mathrm{OH}$ and $v \mathrm{NH}$ and the concentration of $\mathrm{P}_{1}$ had little effect on these peaks, thereby indicating possibility of intramolecular hydrogen within $\mathrm{P} 1$ and intermolecular hydrogen bond among solvent molecules. The $v \mathrm{C}-\mathrm{Cl}, v \mathrm{C}-\mathrm{N}$ and $v \mathrm{C}=\mathrm{O}$ remain 
unchanged after $20 \% \mathrm{ME}$. The aldehyde $\mathrm{vC}-\mathrm{H}$ increased with increase in the polarity media (30 - $90 \% \mathrm{ME}$ ).From the close observation of the Table 6, the UV absorption band of $\mathrm{P}_{1}$ in pure polar aprotic solvent $(\lambda \max =314 \mathrm{~nm}$ in DMSO and $\lambda \max =317$ $\mathrm{nm}$ in DMF) is higher than in polar protic solvent $(\lambda \max =309 \mathrm{~nm})$ except the magnitude of absorbance. The dielectric constant of DMSO is high; therefore there will be strong $\mathrm{P}_{1}$-DMSO interaction. As the $\%$ of $\mathrm{ME}$ is increased, the blue shifts were observed. The wave length of maximum absorption did not lead to significant decrease. Hence, it is the $\pi \rightarrow \pi^{*}$ excitation which corresponds to $\lambda \max =220-230$ $\mathrm{nm}$. This is the evidence for complex formation of $\mathrm{P}_{1}$ with ME through lone pair of electrons on nitrogen as well as oxygen of DMSO and hydrogen of ME. In pure $\mathrm{DMSO} / \mathrm{DMF}$, no such possibility is expected and hence absorbed at higher $\lambda$ max. The decrease was more in ME + DMSO than in ME + DMF. The $\lambda$ max order for $\mathrm{P}_{1}$ in any composition of ME follows $\mathrm{P}_{1}+\mathrm{ME}+\mathrm{DMF}>\mathrm{P}_{1}+\mathrm{ME}+\mathrm{DMSO}$.

Table 4: Selected IR stretching vibrations of three component system P1 + ME + DMSO

\begin{tabular}{|c|c|c|c|c|c|c|}
\hline \multirow{2}{*}{$\%$ ME } & \multicolumn{2}{|c|}{$v$ OH / vNH } & \multicolumn{2}{c|}{$v$ S=O } & \multicolumn{2}{c|}{$v$ C-Cl } \\
\cline { 2 - 7 } & $\mathbf{0 . 0 1 m}$ & $\mathbf{0 . 0 0 1 m}$ & $\mathbf{0 . 0 1 m}$ & $\mathbf{0 . 0 0 1 m}$ & $\mathbf{0 . 0 1 m}$ & $\mathbf{0 . 0 0 1 m}$ \\
\hline 0 & 3446.91 & 3446.91 & 1057.03 & 1053.17 & 698.25 & 698.25 \\
\hline 10 & 3379.4 & 3377.47 & 1057.03 & 1057.03 & 698.25 & 698.25 \\
\hline 20 & 3387.11 & 3385.18 & 1031.95 & 1057.03 & 700.18 & 700.18 \\
\hline 30 & 3389.04 & 3385.18 & 1031.95 & 1031.95 & 700.18 & 702.11 \\
\hline 40 & 3396.76 & 3387.11 & 1030.02 & 1031.95 & 702.11 & 704.04 \\
\hline 50 & 3383.26 & 3377.47 & 1030.02 & 1030.02 & 702.11 & 704.04 \\
\hline 60 & 3379.4 & 3363.97 & 1030.02 & 1030.02 & 704.04 & 704.04 \\
\hline 70 & 3362.04 & 3362.04 & 1030.02 & 1030.02 & 704.04 & 705.97 \\
\hline 80 & 3360.11 & 3352.39 & 1030.02 & 1030.02 & 704.04 & 705.97 \\
\hline 90 & 3358.18 & 3344.68, & 1030.02 & 1030.02 & 704.04 & 705.97 \\
\hline 100 & 3356.25 & 3350.46, & 1030.02 & 1030.02 & 673.18 & 659.68 \\
\hline & & 3333.10 & & & & \\
\hline
\end{tabular}


Table 5: An IR data of groups for $\mathrm{P}_{1}(0.01$ and $0.001 \mathrm{~m})+\mathrm{ME}+\mathrm{DMF}$

\begin{tabular}{|c|c|c|c|c|c|c|c|c|c|c|}
\hline \multirow[t]{2}{*}{$\begin{array}{c}\% \\
\mathrm{ME}\end{array}$} & \multicolumn{2}{|c|}{ vOH } & \multicolumn{2}{|c|}{$\begin{array}{c}v \mathrm{C}=0 \\
\text { (Aldehyde) } \\
\delta \mathrm{N}-\mathrm{H}\end{array}$} & \multicolumn{2}{|c|}{$\begin{array}{c}\text { vC-H } \\
\text { (Aldehyde / } \\
\text { aromatic) }\end{array}$} & \multicolumn{2}{|c|}{$v \mathrm{C}-\mathrm{Cl}$} & \multicolumn{2}{|c|}{$v C-N$} \\
\hline & $0.01 \mathrm{~m}$ & $0.001 \mathrm{~m}$ & $0.01 \mathrm{~m}$ & $0.001 \mathrm{~m}$ & $0.01 \mathrm{~m}$ & $0.001 \mathrm{~m}$ & $0.01 \mathrm{~m}$ & $0.001 \mathrm{~m}$ & $0.01 \mathrm{~m}$ & $0.001 \mathrm{~m}$ \\
\hline 0 & -- & -- & $\begin{array}{l}1689.7, \\
1674.27\end{array}$ & $\begin{array}{l}1668.48, \\
1658.84\end{array}$ & 2929.97 & 2929.97 & 659.68 & 657.75 & 1093.97 & 1093.97 \\
\hline 10 & 3448.84 & 3444.98 & 1674.27 & 1668.48 & 2931.90 & 2931.90 & 659.68 & 659.68 & 1093.97 & 1093.97 \\
\hline 20 & 3433.41 & $\begin{array}{l}3446.91, \\
3433.41\end{array}$ & 1672.34 & 1672.34 & 2933.83 & 2933.83 & 659.68 & 659.68 & 1093.97 & 1093.97 \\
\hline 30 & 3429.55 & 3431.48 & 1668.48 & 1668.48 & 2935.76 & 2935.76 & 661.61 & 661.61 & 1095.60 & 1095.60 \\
\hline 40 & 3425.69 & 3423.76 & 1668.48 & 1668.48 & 2935.76 & 2937.68 & 661.61 & 661.61 & 1095.60 & 1097.53 \\
\hline 50 & 3408.33 & 3412.19 & 1668.48 & 1668.48 & 2939.61 & 2937.68 & 661.61 & 661.61 & 1099.46 & 1097.53 \\
\hline 60 & 3390.97 & $\begin{array}{l}3379.40, \\
3367.82\end{array}$ & 1668.48 & 1668.48 & 2939.61 & 2939.61 & 663.53 & 661.61 & 1099.46 & 1099.46 \\
\hline 70 & $\begin{array}{l}3367.82, \\
3350.46\end{array}$ & $\begin{array}{l}3365.90, \\
3354.32\end{array}$ & 1668.48 & 1668.48 & 2941.54 & 2941.54 & 661.61 & 663.53 & 1099.46 & 1099.46 \\
\hline 80 & 3350.46 & 3352.39 & 1668.48 & 1668.48 & 2943.47 & 2943.47 & 663.53 & 663.53 & 1101.39 & 1101.39 \\
\hline 90 & $\begin{array}{l}3348.54, \\
3333.10\end{array}$ & 3350.46 & 1668.48 & 1668.48 & 2943.47 & 2943.47 & 663.53 & 663.53 & 1101.39 & 1101.39 \\
\hline 100 & 3348.54 & $\begin{array}{l}3346.61, \\
3333.10\end{array}$ & 1666.55 & -- & $\begin{array}{l}2943.47 \\
\text { Alcoholic }\end{array}$ & $\begin{array}{c}2943.47 \\
\text { Alcoholic }\end{array}$ & 659.68 & $\begin{array}{l}675.11, \\
651.96\end{array}$ & 1114.89 & 1114.89 \\
\hline
\end{tabular}

Figure 7 displays minimum energy structures for $\mathrm{P}_{1}+\mathrm{ME}+$ DMSO using Windows based Gaussian 03 and Gauss View 4.1 software. We used Density Functional Theory at 6-31G (d) basis set. The 1:1:1 complex shows presence of both intra and intermolecular hydrogen bonds. ME forms hydrogen bond with oxygen of DMSO. The same oxygen of ME forms hydrogen bond with N-H of $\mathrm{P}_{2}$. 
Table 6: Maximum values of UV wavelength $\left(\lambda_{\max }\right)$ and absorbance for P1 in solvent systems

\begin{tabular}{|c|c|c|c|}
\hline SYSTEM & $\%$ ME & $\lambda_{\max }$ & Absorbance \\
\hline \multirow{5}{*}{ ME+ DMSO } & 0 & 314 & 1.06 \\
\cline { 2 - 4 } & 20 & 234.5 & 3.39 \\
\cline { 2 - 4 } & 40 & 230.5 & 2.88 \\
\cline { 2 - 4 } & 60 & 232.5 & 3.09 \\
\cline { 2 - 4 } & 80 & 228 & 2.82 \\
\cline { 2 - 4 } & 100 & 309 & 1.19 \\
\hline \multirow{5}{*}{ ME + DMF } & 0 & 317 & 2.01 \\
\cline { 2 - 4 } & 20 & 237 & 3.33 \\
\cline { 2 - 4 } & 40 & 232 & 2.99 \\
\cline { 2 - 4 } & 60 & 233.5 & 2.91 \\
\cline { 2 - 4 } & 80 & 232 & 2.84 \\
\cline { 2 - 4 } & 100 & 309 & 1.196 \\
\hline
\end{tabular}

The formation of 3-centered hydrogen bond takes place amongst $\mathrm{N}-\mathrm{H}$ hydrogen of $\mathrm{P}_{1}$ and DMSO methyl hydrogen with nitrogen of $\mathrm{C}=\mathrm{N}$ of aromatic ring $\left(\mathrm{P}_{1}\right)$. The minimum energy structures of $\mathrm{P}_{1}+\mathrm{ME}+\mathrm{DMF}$ are pictured in figure 8. A 3-centered hydrogen bond formation takes place between oxygen of DMF and hydrogen of methyl of ME and ring N-H and hydroxyl group of ME is free. These figures clarified that doublets of $-\mathrm{OH}$ and $-\mathrm{NH}$ groups is due to the proton exchange between $\mathrm{NH}$ of $\mathrm{P}_{1}$ and $\mathrm{OH}$ of $\mathrm{ME}$ in ME rich region of ME + DMSO. Also, the theoretical study helped us to confirm our conclusions drawn from UV data of this ternary system.

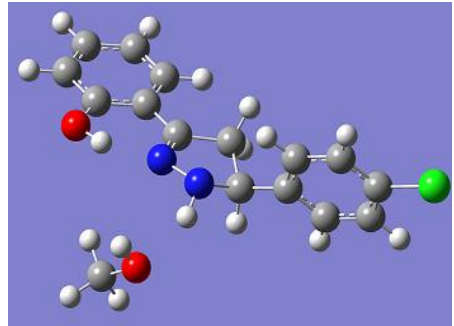

(a)

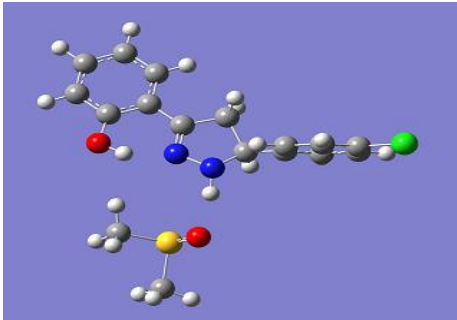

(b)

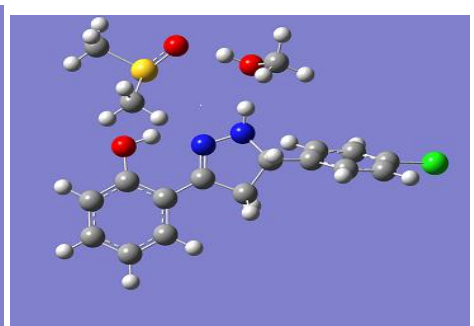

(c)

Figure 7: (a) DFT minimum energy diagrams of $\mathrm{P}_{1}+\mathrm{ME}$ complexes using B3LYP/6-31G(d) basis set. (b) DFT minimum energy diagrams of $\mathrm{P}_{1}+$ DMSO complexes using B3LYP/6-31G(d) basis set. (c) DFT minimum energy diagrams of $\mathrm{P}_{1}+\mathrm{ME}+\mathrm{DMSO}$ complexes using B3LYP/6-31G(d) basis set 


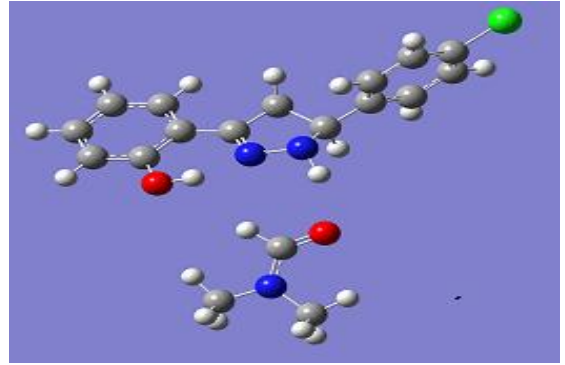

(a)

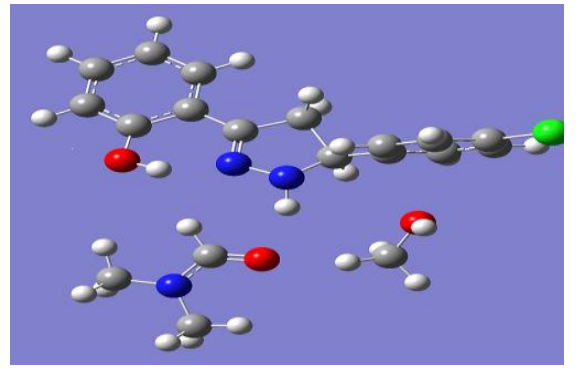

(b)

Figure 8: (a) DFT Optimized geometries of 1:1 and 1:1:1 complexes of $\mathrm{P}_{1}$ with DMF using B3LYP/6-31G(d) basis set (b) DFT Optimized geometries of 1:1 and 1:1:1 complexes of $\mathrm{P}_{1}$ with ME and DMF using B3LYP/6-31G(d) basis set

\section{CONCLUSION}

Hydrogen bonding plays an important role in both fundamental science and industrial applications. IR spectroscopy is one of the methods for identification of hydrogen bonds. UV spectroscopy deals with the excitation of non and pi bonding electrons.

An irregular trends in $v \mathrm{OH}, v \mathrm{C}=\mathrm{O}, v \mathrm{~S}=\mathrm{O}, v \mathrm{C}-\mathrm{Cl}, v \mathrm{C}-\mathrm{H}, v \mathrm{NH}$ etc .with increase of $\mathrm{ME}$ in solution at a particular concentration of solute indicated structure of the medium changed with ME content. The $\lambda$ max of solutes vary with the $\%$ of ME. The decrease in $\lambda \max$ of all solutes was more in ME + DMSO than in ME + DMF. The amount of blue shift (lower wave length) has been used as a measure to the strength of hydrogen bond. Absence of $\mathrm{n} \rightarrow \pi^{*}$ excitation in solute $+\mathrm{ME}+\mathrm{DMSO} / \mathrm{DMF}$ was ascribed due to hydrogen bond formation.

Theoretical quantum calculations of these systems using GO3 (W) software improved our knowledge of kinds of intermolecular interactions taking place in systems. In this dissertation, we are giving the optimized geometries only. For this purpose Gauss View 4.1 package was used.

From the optimized geometries of $\mathrm{P}_{1}$ in $\mathrm{ME}+\mathrm{DMSO} / \mathrm{DMF}$ we conclude that ME forms hydrogen bond with $\mathrm{N}-\mathrm{H} / \mathrm{C}=\mathrm{O}$ of solute The oxygen of $\mathrm{ME}$ was very close to sulphur atom of DMSO showing dipole-dipole interactions between them. Very interesting result of this study was the formation of 3-centered hydrogen bond between two hydrogen atoms of aromatic rings of solute and sulphur atom of DMSO.

Distinguish between $v \mathrm{OH}$ and $v \mathrm{NH}$ is generally difficult because these frequencies appear in the same region $\left(3500-3300 \mathrm{~cm}^{-1}\right)$. With Density Functional Theory at 6$31 \mathrm{G}$ (d) basis set, the 1:1:1 complex showed presence of both intra and intermolecular hydrogen bonds for $\mathrm{P}_{1}$. ME formed hydrogen bond with oxygen of DMSO. The same oxygen of ME formed hydrogen bond with $\mathrm{N}-\mathrm{H}$ of $\mathrm{P}_{1}$. The formation of 3-centered hydrogen bond takes place amongst $\mathrm{N}-\mathrm{H}$ hydrogen of $\mathrm{P}_{1}$ and DMSO methyl hydrogen with nitrogen of $\mathrm{C}=\mathrm{N}$ of aromatic ring $\left(\mathrm{P}_{1}\right)$. 


\section{ACKNOWLEDGEMENT}

The author is thankful to Hon. Members of the management committee, Pimpalner education society, Pimpalner and also to the Principal of the College for providing facilities. My sincere thank and appreciation to Arunkumar B. Sawant, Ex. Professor in Chemistry, M. S. G. College, Malegaon Camp (Nashik), for his valuable and masterly guidance

\section{REFERENCES}

[1] Jamode V S,Chandak H S \& Bhagat P R, Asian J Chem, 16 (2004) 233; Chem Abstr, 141 (2004) 243402s.

[2] Koregaokar S S, Patil P H, Shah M T \& Parekh H H, Indian J Pharm Sci, 58 (1996) 222.

[3] Babu V H, Manna S K, Sneha, Srinivasan K K \& Bhat G V, Indian J Hetercycl Chem, 13 (2004) 253; Chem Abstr, 141 (2004) 314227b.

[4] Taylor E C, Patel H \& Kumar H, Tetrahedron, 48 (1992) 8089.

[5] Ruhoglu O, Ozdemir Z, Calis U, Gumusel B \& Bilgin A A, Arzneim Foresch, 55, (2005) 431.

[6] Chauhan R K, Yoon S J, Lee H, Kang M C and Min B M ; J Chem Eng Data,48 (2003) 291.

[7] Forkmann G and Heller W, "Comprehensive Natural Products Chemistry", Barton D, Nakanishiv K, Meth-Cohn O (Eds.) (Elservier Science, Amsterdam), (1999)713.

[8] Agrawal N N and Soni P A; Indian J Chem, 44B (2005) 2601. a) Geissman T A and Harborne J B ; J Am Chem Soc , 78 (956) 832. b) Harbrone J B,

[9] Rao Y J, Reddy C R, Gangadhar N and David Krupadanam G L ; Indian J Chem, 48B (2009) 1329.

[10] Mahadevan K M, Basavaraj K M, Prathima Mathias D A and Vaidya V P, Indian J Chem, 44B (2005) 789.

[11] El-Sawy, Shaker K H, Mandour A H, El-Din A S and Abdula M M, Indian J Chem, 47 (2008) 1451.

[12] Karabasanagouda T, Airody V A and Girisha M; Indian J Chem, 48B (2009) 430.

[13] Hasan M, Kadam U B, Hiray A P and Sawant A B ; J Chem Eng Data,51 (2006) 1797.

[14] Davis, B.J., Carney, P.S. \& Bhargava, R. Theory of mid-infrared absorption micro spectroscopy: II. Heterogeneous samples. Anal. Chem. 82, 3487-3499 (2010).

[15] Frisch M J et al Gaussian 03, Revision E01 Gaussian, Inc., Wallingford CT, USA 2004. 
